\title{
BOUSSINESQ MODELLING OF SOLITARY WAVE PROPAGATION, BREAKING, RUNUP AND OVERTOPPING
}

\author{
Jana Orszaghova ${ }^{1}$, Alistair G. L. Borthwick ${ }^{1}$, Paul H. Taylor ${ }^{1}$
}

\begin{abstract}
A one-dimensional hybrid numerical model is presented of a shallow-water flume with an incorporated piston paddle. The hybrid model is based on the improved Boussinesq equations by Madsen and Sørensen (1992) and the nonlinear shallow water equations. It is suitable for breaking and non-breaking waves and requires only two adjustable parameters: a friction coefficient and a wave breaking parameter. The applicability of the model is demonstrated by simulating laboratory experiments of solitary waves involving runup at a plane beach and overtopping of a laboratory seawall. The predicted free surface profiles, maximum runup and overtopping volumes agree very well with the measured values.
\end{abstract}

Keywords: Boussinesq equations, numerical modelling, runup, overtopping, solitary waves

\section{INTRODUCTION}

Numerical models based on Boussinesq-type equations represent a practical and accurate choice for simulations of nearshore hydrodynamic processes. Their simplicity, due to the explicit elimination of the vertical coordinate, significantly reduces the computational time. Yet the inclusion of nonlinear and dispersive terms to different orders depending on the particular equation set, allows for a realistic representation of wave shoaling, dispersion and propagation.

Since the classical Boussinesq equation set derived by Peregrine (1967), which retains first order nonlinearity and first order dispersion, there has been considerable progress made in extending the applicability of Boussinesq equations both offshore and onshore. Improvements to the dispersion properties, notably by Madsen and Sørensen (1992), Nwogu (1993) and Schäffer and Madsen (1995), and derivations of higher order equation sets such as Gobbi et al. (2000) and Madsen et al. (2003) have made it possible to apply Boussinesq-type equations in water depths as deep as $\frac{h}{L}=6$ where $h$ is the still water depth and $L$ is the wavelength. By inclusion of a wave breaking threshold criterion and an energy dissipation mechanism, wave breaking has been succesfully simulated within the framework of Boussinesq solvers. This has enabled the application of Boussinesq-type equations all the way inshore including the surf and swash zones. The most widely used approaches for wave breaking treatment have been the inclusion of eddy viscosity, the surface roller concept and the vorticity transport models.

The non-linear shallow water equations have been used extensively for simulations of shallow flows involving bores, wave runup and overtopping, see for example Hubbard and Dodd (2002), Brufau et al. (2002) and Liang and Borthwick (2009). Since the equations are non-dispersive, they cannot accurately model wave transformations which occur before breaking and as such their use in coastal wave modelling should be restricted to broken waves and very shallow depths where $\frac{h}{L}<0.05$.

The present paper describes a hybrid numerical model with two sets of governing equations, the improved Boussinesq equations by Madsen and Sørensen (1992) and the non-linear shallow water equations. The Boussinesq equations are used pre-breaking and the shallow water equations post-breaking. The breaking initiation criterion is based on the local wave steepness. Energy dissipation and loss of wave height are naturally handled by the shallow water equations. This simple, yet very effective, breaking treatment requires only one adjustable parameter. Similar hybrid models have been proposed by Weston et al. (2004), Tonelli and Petti (2009) and Bonneton et al. (2010). The model contains a wetting and drying algorithm and therefore can be used to simulate wave runup and rundown as well as an overtopping event when the water mass might separate into disjoint water bodies. A novel feature of the present model is an integrated piston paddle wavemaker. It operates in the same way as a laboratory piston paddle by moving according to a supplied paddle displacement signal. The issue of the moving paddle surface has been overcome by implementing a time varying local grid which is fully Lagrangian at the paddle face and Eulerian within the bulk of the domain. Our present model is effectively a complete numerical flume suitable for shallow water experiments. The applicability of the

\footnotetext{
${ }^{1}$ Department of Engineering Science, University of Oxford, Parks Road, Oxford, OX1 3PJ, UK
} 
model is demonstrated by simulating runup and overtopping laboratory experiments of solitary waves.

\section{THE NUMERICAL MODEL}

In this section we present the numerical model, starting with the governing equations. Particular attention is given to the treatment of wave breaking, the piston paddle wavemaker, the wetting and drying algorithm, and boundary conditions.

The governing equations of the hybrid model comprise the enhanced Boussinesq equations by Madsen and Sørensen (1992) and the nonlinear shallow water equations. We adopt an $(\eta, q)$ formulation for both equation sets, where $\eta$ denotes the water surface level above a prescribed datum and $q$ denotes the depth-integrated horizontal flux, see Figure 1.

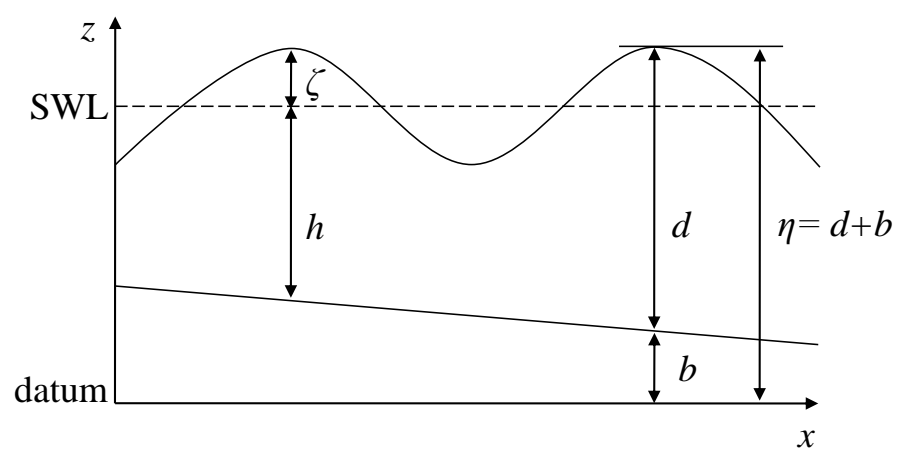

Figure 1: Definition sketch.

\section{Nonlinear Shallow Water Equations}

The one-dimensional nonlinear shallow water equations, describing conservation of mass and horizontal linear momentum, read respectively as

$$
\begin{aligned}
\eta_{t}+q_{x} & =0 \\
q_{t}+\left(\frac{q^{2}}{d}+\frac{1}{2} g\left(\eta^{2}-2 \eta b\right)\right)_{x} & =-\frac{\tau_{b}}{\rho}-g \eta b_{x}
\end{aligned}
$$

where $t$ is time, $x$ is horizontal distance, $d$ is the local water depth, $b$ is the bed elevation, $\tau_{b}$ is the bed frictional stress, $\rho$ is the water density, and $g$ is the acceleration due to gravity. Subscript notation is used for partial differentiation.

Equations (1) and (2) are well suited for finite volume solvers, as they form a hyperbolic set of equations, $\boldsymbol{q}_{t}+\boldsymbol{f}(\boldsymbol{q})_{x}=\boldsymbol{s}$, with $\boldsymbol{q}$ representing the vector of conserved variables $(\eta, q)^{-1}, \boldsymbol{f}$ the vector of fluxes, and $s$ the vector of source terms. In the present model, the integral form of the above equation system (1) and (2) is solved using a Godunov-type shock-capturing scheme with the HLL approximate Riemann solver for calculation of the intercell fluxes. MUSCL-Hancock data reconstruction and predictor-corrector time-stepping lead to second order accuracy of the method in time and space. A fixed regular grid of spacing $\Delta x$ is used. More details of the solver can be found in Liang and Borthwick (2009).

Wetting and drying at the moving shoreline is calculated as part of the solution by means of a technique proposed by Brufau et al. (2002) and the dry-bed extension of the HLL solver, see Fraccarollo and Toro (1995). Dry cells are excluded from the computational domain unless they are about to be flooded. At each wetting/drying front, local bed modification in fictitious cells is carried out as a neat way of implementing boundary conditions.

\section{Boussinesq equations}

The improved Boussinesq equations by Madsen and Sørensen (1992) contain additional dispersive terms in the momentum equation, when compared to the nonlinear shallow water equa- 
tions. The equations are recast for numerical convenience as follows,

$$
\begin{aligned}
\eta_{t}= & -q_{x} \\
q_{t}^{*}= & -\left(\frac{q^{2}}{d}+\frac{1}{2} g\left(\eta^{2}-2 \eta b\right)\right)_{x}-\frac{\tau_{b}}{\rho}-g \eta b_{x}+ \\
& +B g h^{3} \eta_{x x x}-2 B g h^{2} b_{x} \eta_{x x}
\end{aligned}
$$

where

$$
q^{*}=q-\left(B+\frac{1}{3}\right) h^{2} q_{x x}+\frac{1}{3} h b_{x} q_{x}
$$

In the above equations $B$ represents the Boussinesq linear dispersion enhancement coefficient. Setting $B=\frac{1}{15}$ embeds the [2][2] Padé approximant of the linear dispersion relation into the momentum equation (4). Setting $B=0$ implies no improvement of the linear dispersion properties and reverts back to the classical Boussinesq equations originally derived by Peregrine (1967).

Equations (3), (4) with (5) are solved using finite differencing. Spatial derivatives are approximated by 5 -point centered stencils leading to overall second order accuracy. As pointed out by Wei and Kirby (1995), this ensures that the truncation error arising from approximating first order derivatives is smaller than the third order derivative dispersive terms in the momentum equations (4). The equations are integrated forward in time using a fourth order Runge-Kutta method for a sytem of equations. At each of the 4 intermediate steps in the Runge-Kutta procedure, equation (5) is solved for $q$. When discretised, it forms a pentadiagonal system, which is efficiently solved by an extended Thomas algorithm.

In order to incorporate a piston paddle moving boundary into the model, a moving grid is used in the vicinity of the paddle. The grid adapts according to the desired paddle position from the paddle displacement signal fed into the model. When the paddle moves forwards, to create a wave crest, the moving grid contracts. When the paddle moves backwards, to create a wave trough, the moving grid expands. Boundary conditions at the paddle are handled by the use of ficticious cells and the known paddle displacement signal. The updated value of $\eta$ on the paddle is calculated as part of the solution. The updated value of $q$ on the paddle is calculated from the current paddle velocity which is derived from the supplied paddle displacement signal. Full details of implementation of the numerical paddle, including verification examples, are to be given in a paper in preparation by Orszaghova et al. (2011).

\section{Wave Breaking}

The present hybrid model is suitable for breaking and non-breaking waves. Prior to breaking, the Boussinesq equations (3),(4) and (5) are used. Broken waves are modelled as bores by the nonlinear shallow water equations (1) and (2). The nonlinear shallow water equations are a subset of the Boussinesq equations and thus switching between the two sets of equations is a matter of retaining or neglecting the dispersive terms. To reduce any disturbances in the flow, the dispersive terms are ramped down smoothly.

The breaking criterion is based on the local wave slope $\eta_{x}$. At every timestep, the domain is separated into individual waves using a down-crossing method. Wave steepness $\eta_{x}$ is checked for each wave. Waves with $\eta_{x} \geq 0.4$ are assumed to be breaking and thus their flow is governed by the shallow water equations leading to further steeping and bore formation. The shallowness parameter $\mu=\frac{h}{L}$ is also checked. In very shallow waters with $\mu<0.05$, the shallow water equations are applied since dispersion is assumed to be negligible.

\section{SIMULATION RESULTS}

In this section, the model is validated for solitary wave runup on a plane beach and overtopping of a laboratory sea-wall, allowing comparisons with small and large scale experiments.

\section{Solitary wave runup at a plane beach}

Synolakis (1987) performed high quality experimental measurements of solitary wave runup at a plane beach at laboratory scale. The flume was $40 \mathrm{~m}$ long, and contained a plane beach of slope $1: 19.85$. By varying the amplitude of the solitary wave $A$ and the water depth at the paddle $h_{0}$, Synolakis was able to collect data on runup for a wide range of input solitary wave conditions, covering $0.005 \leq \epsilon \leq 0.633$, where $\epsilon=\frac{A}{h_{0}}$ represents the solitary wave nonlinearity. 
The present model has been used to reproduce several of Synolakis' cases, but covering the entire range. The bed friction coefficient is set to $C_{f}=0.0056$ in all cases. The grid spacing of $\Delta x=4 \mathrm{~cm}$ and the time step of $\Delta t=0.005 \mathrm{~s}$ were used. Figure 2 is a plot of the nondimensional runup against non-dimensional solitary wave amplitude, on which is shown both Synolakis' experimental results and the predictions by the present model. There is excellent agreement between the numerical predictions and experimental measurements.

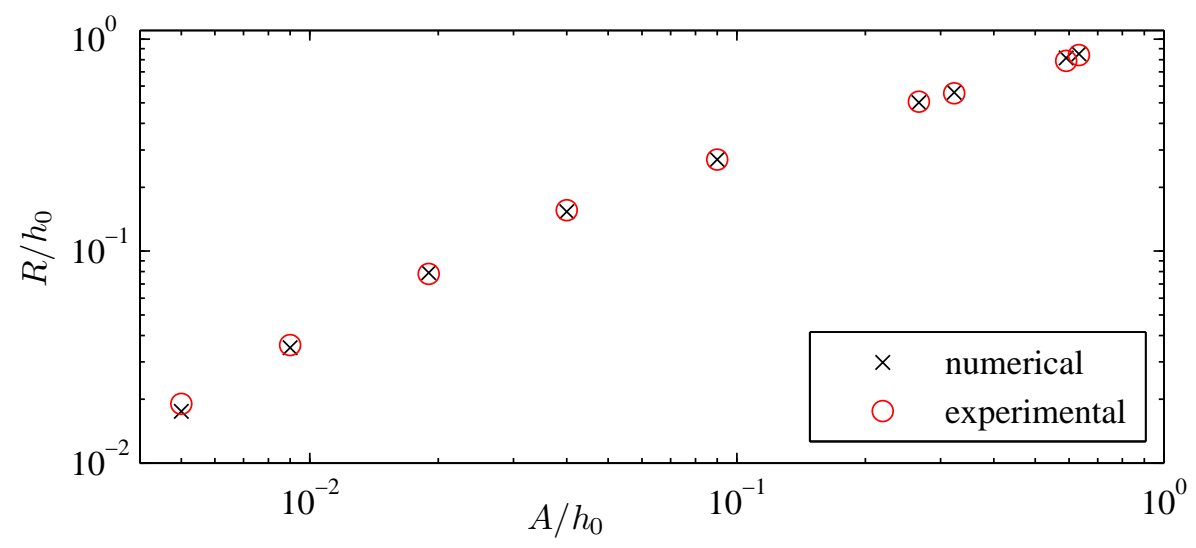

Figure 2: Non-dimensionalised runup. Comparison between the numerical predictions and the experimental data of Synolakis (1987).

More recently, Hsiao et al. (2008) carried out large scale laboratory experiments of the evolution of breaking solitary waves on a plane $1: 60$ beach. The supertank used in the experiments was $300 \mathrm{~m}$ long and $5 \mathrm{~m}$ wide. The toe of the beach was $50 \mathrm{~m}$ from the wavegenerator, as shown in Figure 3. The free surface elevation was measured at over 80 locations along the tank, at a sampling rate of $50 \mathrm{~Hz}$. The gauge at $x=24 \mathrm{~m}$ acted as the reference wave gauge where the offshore amplitude $A$ was measured. The maximum runup heights were obtained from visual observations and runup sensor.

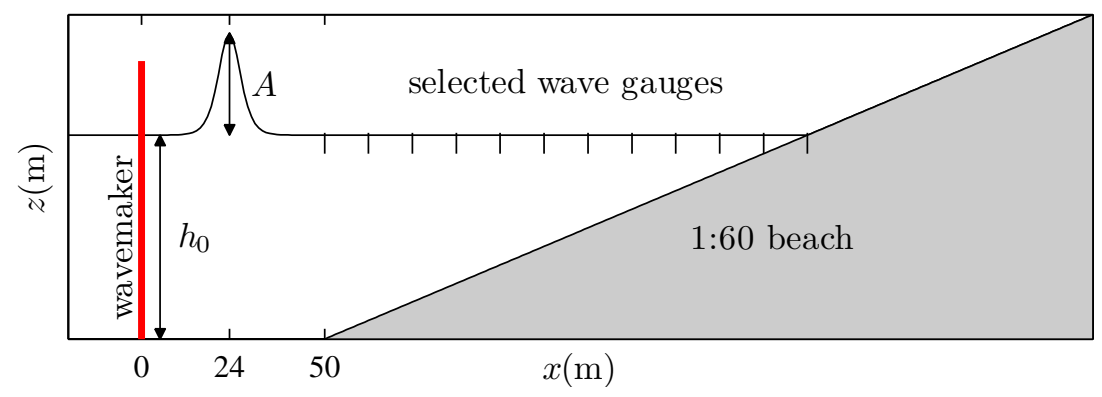

Figure 3: Definition sketch for the experiments of Hsiao et al. (2008).

The present model has been used to simulate 6 experimental runs, with the solitary wave nonlinearity $\epsilon$ ranging from 0.054 to 0.338 . The chosen cases are summarised in Table 1 below.

Table 1: Experimental and numerical data for the chosen cases of Hsiao et al. (2008).

\begin{tabular}{|l|l|l|l|l|}
\hline Trial & $h_{0}(\mathrm{~m})$ & $\epsilon$ & $R / h_{0}$ measured & $R / h_{0}$ predicted \\
\hline 12 & 1.2 & 0.208 & 0.208 & 0.2115 \\
16 & 1.2 & 0.264 & 0.236 & 0.2353 \\
19 & 1.2 & 0.338 & 0.261 & 0.2614 \\
25 & 2.2 & 0.054 & 0.111 & 0.1156 \\
35 & 2.2 & 0.120 & 0.165 & 0.1655 \\
43 & 2.2 & 0.152 & 0.180 & 0.1828 \\
\hline
\end{tabular}


In the simulations, the waves were generated by the in-built piston paddle. For Trials 12,16 and 19 the experimental paddle displacement signals were recorded. These were fed into the model, after smoothing and applying a small gain in order to match the wave amplitude at the reference wave gauge. For Trials 25, 35 and 43 the paddle signals were calculated according to Goring (1978). The model was run on a grid with spacing $\Delta x=4 \mathrm{~cm}$, with the moving grid in the vicinity of the paddle automatically adapting according to the current paddle position. The timestep was $\Delta t=0.01 \mathrm{~s}$. It should be noted that the grid resolution and time step have been chosen so that the model gives converged, stable results. The friction coeffient was set to $C_{f}=0.003$. Figure 4 shows the free surface time histories at 12 different gauges along the flume, starting at the beach toe and finishing at the shoreline. For almost all gauge positions, there is remarkable agreement between the numerical predictions and experimental data. The model properly reproduces the propagation of the solitary wave before it reaches the toe of the beach, the steepening of the wave front as it travels up the beach, the breaking event, and subsequent bore production. There is also evidence of the oscillatory trailing wave motion behind the primary soliton for the more nonlinear cases. The predicted bore is slightly larger than the measured at the final gauge for the more non-linear cases. The non-dimensionalised values of the measured and predicted runup are listed in Table 1. The predictions are excellent with maximum error of $4.1 \%$.

\section{Solitary wave overtopping a seawall}

Hunt (2003) and Borthwick et al. (2006) conducted a large series of runup and overtopping experiments in the UK Coastal Research Facility (UKCRF). One such experiment involved a solitary wave of $0.1 \mathrm{~m}$ amplitude being generated in $0.5 \mathrm{~m}$ depth, propagating up a $1: 20$ beach and overtopping a laboratory seawall. The central section of the seawall was flat, the sloping sides had roughly $1: 2$ inclination. For detailed dimensions of the seawall see Hunt (2003). As shown in Figure 5, the toe of the beach was $8.33 \mathrm{~m}$ from the 72 -paddle wavemaker. The toe of the sea wall was $8.125 \mathrm{~m}$ from the toe of the beach. Free surface elevation was measured by 30 wave gauges positioned along the beach, including the seawall. Overtopped volume was measured from a catchment area, positioned behind the seawall, which had a V-notch weir.

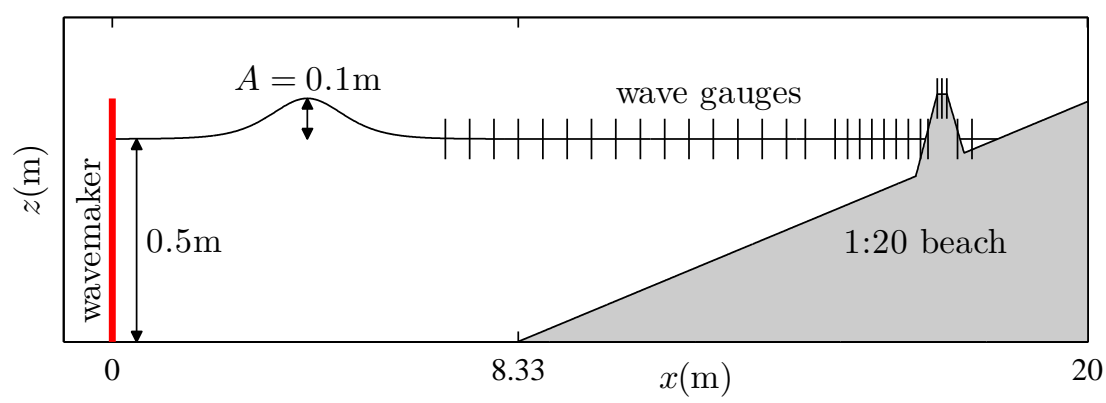

Figure 5: Definition sketch for the experiment of Hunt (2003).

The experiment was reproduced with the present hybrid model. The solitary wave was generated using the identical paddle displacement signal that was fed into the UKCRF wavemaker during the experiment. The computational grid had spatial resolution of $\Delta x=1 \mathrm{~cm}$ and the timestep was $\Delta t=0.005 \mathrm{~s}$. The friction coefficient was set to $C_{f}=0.008$. Figure 6 shows the predicted and measured free surface spatial profiles of the solitary wave at times $t=9,10,11$, 12 and $13 \mathrm{~s}$ after the start of the wave generation. There is very good agreement throughout the whole process. Wave steepening and breaking, as well as overtopping the seawall and subsequent rundown are properly resolved by the model. The amount of overtopped water was also calculated by the model. There was roughly a $12 \%$ mismatch, with the predicted value of 34.1 $\mathrm{l} / \mathrm{m}$ and the measured volume of $30.5 \mathrm{l} / \mathrm{m}$. 


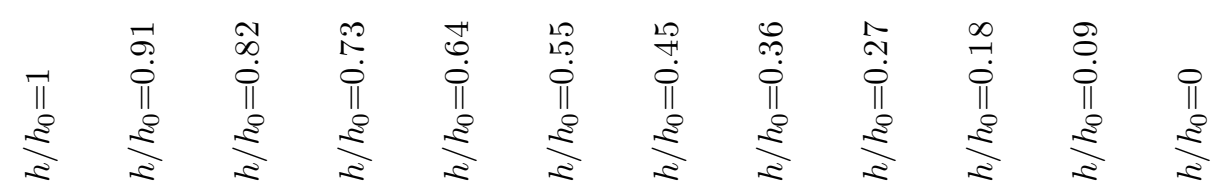
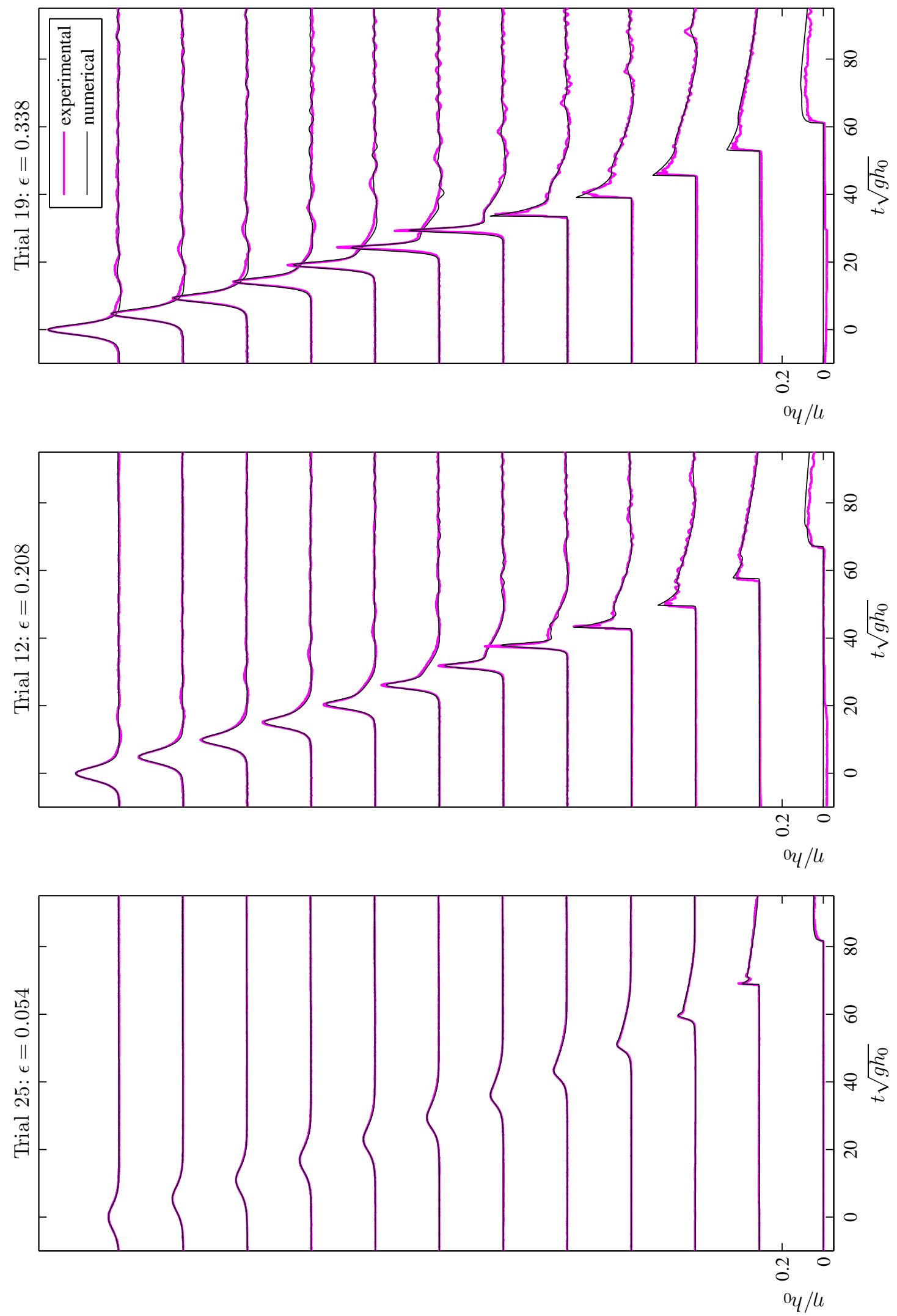

Figure 4: Non-dimensionalised free surface elevation time series. Comparison between the numerical predictions and the experimental data from Hsiao et al. (2008). 

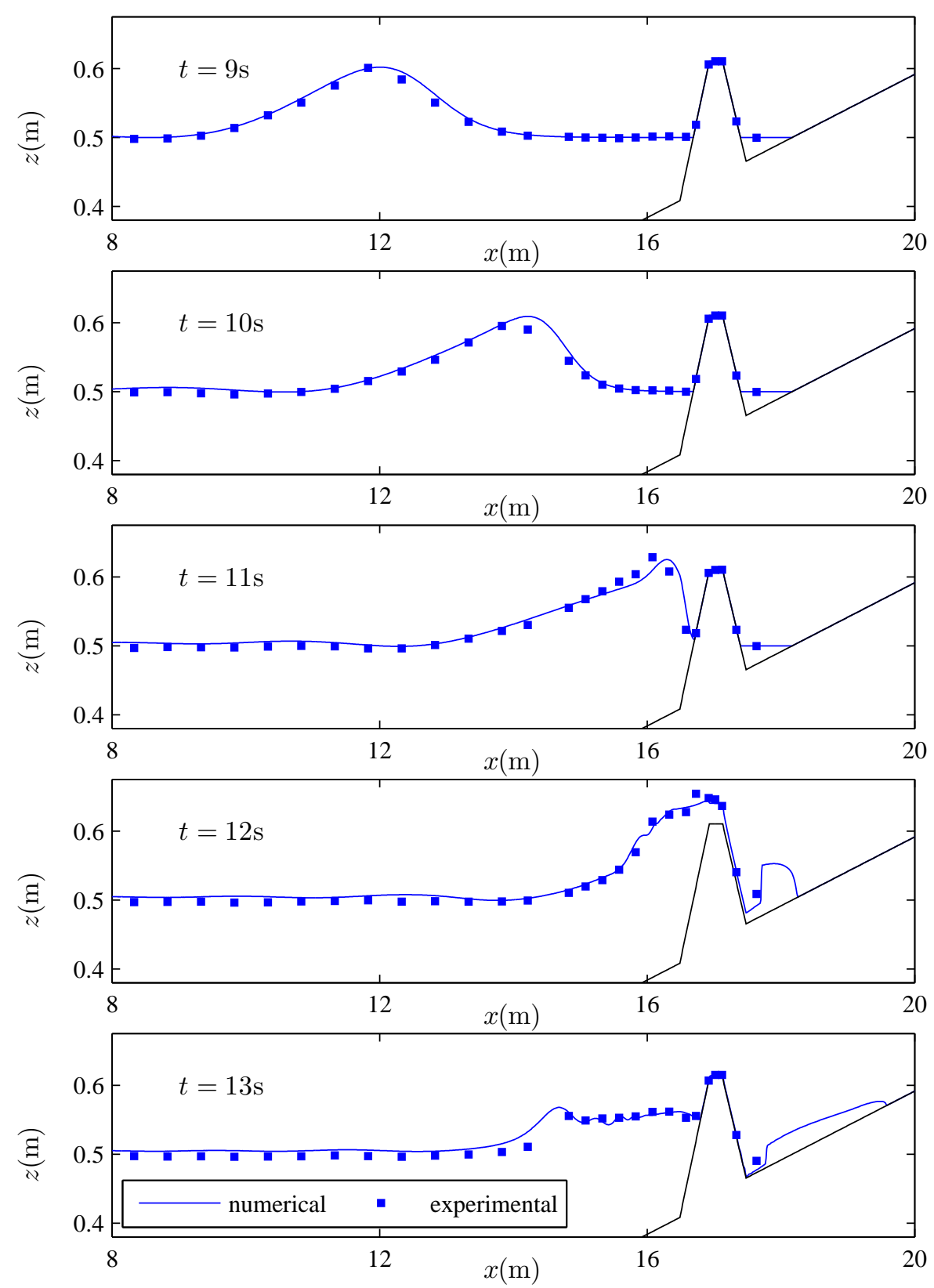

Figure 6: Spatial profiles of the overtopping solitary wave. Comparison between the numerical predictions and the experimental data from Hunt (2003).

\section{CONCLUSIONS}

A hybrid numerical model has been developed based on the Boussinesq-type equations of Madsen and Sørensen (1992) and the nonlinear shallow water equations. Particular care has been taken to ensure that the breaking process is reasonably smooth, with regard to the switch between the full Boussinesq-type equations and the nonlinear shallow water equations. The model includes a wetting and drying scheme based on that of Brufau et al. (2002), which appears to work well with regard to solitary wave runup and rundown. Inclusion of a piston paddle moving boundary in the model means that the driving signal for the numerical paddle is essentially that which would be sent to the paddle in practice. The model has been successfully validated using three sets of experiments, involving solitary wave runup on a plane beach and solitary wave overtopping a laboratory seawall. There is striking agreement between the predicted and experimental data in terms of maximum runup values and free surface elevation throughout the 
wave evolution. Satisfactory prediction is also found for overtopping volume.

\section{ACKNOWLEDGEMENTS}

The authors would like to thank Prof. Shih-Chun Hsiao and his team from the National Cheng Kung University, Taiwan, and Dr. Alison C. Hunt-Raby from the University of Plymouth, UK and Dr. Tong Feng of Manchester University, UK for providing the experimental data. Financial assistance from the UK Engineering and Physical Sciences Research Council, the Royal Academy of Engineering and Christ Church College, University of Oxford is also gratefully acknowledged.

\section{REFERENCES}

Bonneton, P., Chazel, F., Lannes, D., Marche, F., and Tissier, M. (2010). A splitting approach for the fully nonlinear and weakly dispersive Green-Naghdi model. Journal of Computational Physics, submitted.

Borthwick, A. G., Hunt, A. C., Feng, T., Taylor, P. H., and Stansby, P. K. (2006). Flow kinematics of focused wave groups on a plane beach in the U.K. Coastal Research Facility. Coastal Engineering, 53:1033 - 1044.

Brufau, P., Vázquez-Cendón, M. E., and García-Navarro, P. (2002). A numerical model for the flooding and drying of irregular domains. International Journal for Numerical Methods in Fluids, 39:247 - 275.

Fraccarollo, L. and Toro, E. F. (1995). Experimental and numerical assessment of the shallow water model for two-dimensional dam-break type problems. Journal of Hydraulic Research, 33:843-864.

Gobbi, M. F., Kirby, J. T., and Wei, G. (2000). A fully nonlinear Boussinesq model for surface waves. Part 2. Extension to $\mathrm{O}(\mathrm{kh}) 4$. Journal of Fluid Mechanics, 405:181-210.

Goring, D. G. (1978). Tsunamis The Propagation of Long Waves Onto a Shelf. PhD thesis, Rep. No. KH-R-38, W.M. Keck Laboratory of Hydraulics and Water Resources, California Institute of Technology.

Hsiao, S.-C., Hsu, T.-W., Lin, T.-C., and Chang, Y.-H. (2008). On the evolution and run-up of breaking solitary waves on a mild sloping beach. Coastal Engineering, 55:975 - 988.

Hubbard, M. E. and Dodd, N. (2002). A 2D numerical model of wave run-up and overtopping. Coastal Engineering, 47:1 - 26.

Hunt, A. C. (2003). Extreme Waves, Overtopping and Flooding at Sea Defences. PhD thesis, University of Oxford, UK.

Liang, Q. and Borthwick, A. (2009). Adaptive quadtree simulation of shallow flows with wet-dry fronts over complex topography. Computers \&f Fluids, 38:221 - 234.

Madsen, P. A., Bingham, H. B., and Schäffer, H. A. (2003). Boussinesq-type formulations for fully nonlinear and extremely dispersive water waves: derivation and analysis. Proceedings of the Royal Society A, 459:1075 - 1104.

Madsen, P. A. and Sørensen, O. R. (1992). A new form of the Boussinesq equations with improved linear dispersion characteristics. Part 2. A slowly-varying bathymetry. Coastal Engineering, 18:183 - 204.

Nwogu, O. (1993). Alternative form of Boussinesq equations for nearshore wave propagation. Journal of Waterway, Port, Coastal, and Ocean Engineering, 119:618-638.

Orszaghova, J., Borthwick, A. G. L., and Taylor, P. H. (2011). A hybrid Boussinesq-Shallow Water Equations wavetank for coastal modelling. In preparation.

Peregrine, D. H. (1967). Long waves on a beach. Journal of Fluid Mechanics, 27:815-827.

Schäffer, H. A. and Madsen, P. A. (1995). Further enhancements of Boussinesq-type equations. Coastal Engineering, 26:1 - 14.

Synolakis, C. E. (1987). The runup of solitary waves. Journal of Fluid Mechanics, 185:523-545.

Tonelli, M. and Petti, M. (2009). Hybrid finite volume - finite difference scheme for 2DH improved Boussinesq equations. Coastal Engineering, 56:609 - 620 .

Wei, G. and Kirby, J. T. (1995). Time-dependent numerical code for extended Boussinesq equations. Journal of Waterway, Port, Coastal, and Ocean Engineering, 121:251-261.

Weston, B. P., Taylor, P. H., Borthwick, A. G. L., and Hunt, A. C. (2004). Godunov-type Boussi- 
nesq modelling of extreme wave run-up. In G. H. Jirka, W. S. J. U., editor, Shallow Flows: Research Presented at the International Symposium on Shallow Flows, Delft, Netherlands, 2003, pages 398-403. Taylor \& Francis. 